\title{
A middle-aged man with indurated scrotal nodules
}

\author{
Diego Fernández-Nieto, \\ Darío de Perosanz-Lobo, \\ Juan Jiménez-Cauhé, \\ Daniel Ortega-Quijano, \\ Pedro Jaén-Olasolo
}

\section{CASE}

A man aged 45 years with no notable medical history presented with a one-year history of asymptomatic scrotal nodules. He had a history of progressively enlarging scrotal lesions with no preceding local trauma. He had no other lesions on the shaft or glans, no urethral or urinary symptoms and he denied a previous history of unsafe sexual practices. Physical examination revealed three whitish/ skin-coloured nodules of $0.5 \mathrm{~cm}$ each without local reaction (Figure 1). A hard, solid, homogenous content was noticeable by touch. Laboratory tests revealed normal phosphorus and calcium levels.

\section{QUESTION 1}

What is the diagnosis?

\section{QUESTION 2}

What are the potential differential diagnoses?

\section{QUESTION 3}

What are the treatment options for this condition?

\section{ANSWER 1}

This condition is known as scrotal calcinosis (formerly idiopathic scrotal calcinosis). ${ }^{1}$ It is a disorder characterised by the progressive appearance of solitary or multiple calcified, painless nodules on the scrotum. The nodules usually appear during early adulthood and tend to increase in size and number with age. They are usually asymptomatic but can be pruritic or result in a heavy sensation or chalky material discharge. ${ }^{2}$

Scrotal calcinosis was considered idiopathic in the past because no association with other lesions or known systemic pre-conditions was observed. It is not related to a calcium phosphate imbalance or renal insufficiency. Infiltration of foreign body material or previous local trauma as predisposing factors remain controversial. ${ }^{3}$

The condition has more recently been attributed to dystrophic calcification of epidermoid cysts. ${ }^{4}$ It occurs as a process of subclinical inflammation, rupture, dystrophic calcification and finally obliteration of the cyst wall. ${ }^{5}$

\section{ANSWER 2}

Scrotal calcinosis is easily recognised if suspected. The patient presented with multiple epidermal cystic lesions characterised by their hard homogenous content, with a solid appearance. Epidermoid carcinoma of the scrotum is very rare and usually manifests as a single verrucous indurated mass. Testicular cancer is deeper and does not have epidermal involvement.

Scrotal calcinosis can be confused or coexist with typical epidermoid cysts located at the scrotum or nearby areas. ${ }^{6}$ It lacks the inflammatory appearance of furuncles or abscesses and rarely develops superinfection.

\section{ANSWER 3}

Scrotal calcinosis is a benign condition, and surgical intervention is only recommended in severe cases with disturbance of scrotal appearance or in the context of associated symptoms. Surgical excision is the main procedure. Because of the laxity of scrotal skin, a single-stage excision can be easily performed for small or medium defects. ${ }^{2}$ A recent novel technique using an erbium:YAG laser has been trialled as a less invasive method. It has good aesthetic and functional results with lower risks of injury and infection. ${ }^{7}$

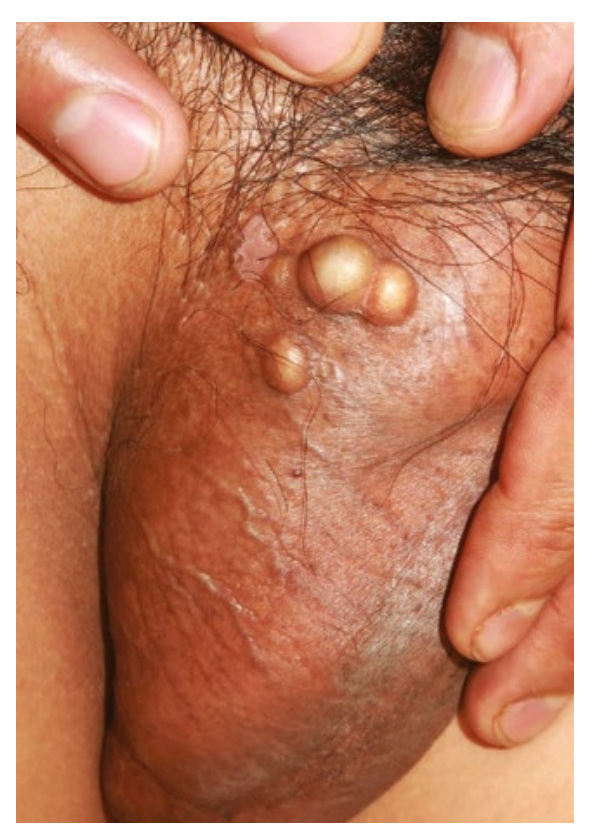

Figure 1. Scrotal calcinosis; three indurated whitish/skin-coloured nodules located at right scrotum 


\section{CASE CONTINUED}

The patient was reassured about the benign nature of his condition. He had no symptoms or aesthetic concerns and it did not interfere with his sex life. No further intervention was required.

\section{Key points}

- Scrotal calcinosis is a benign condition that can be easily recognised if suspected.

- Surgical treatment is only recommended in the context of local symptoms or aesthetic reasons.

\section{Authors}

Diego Fernández-Nieto MD, Dermatologist, Dermatology Department, Hospital Universitario Ramón y Cajal, Madrid, Spain. fnietodiego@gmail.com
Darío de Perosanz-Lobo MD, Dermatologist, Dermatology Department, Hospital Universitario Ramón y Cajal, Madrid, Spain

Juan Jiménez-Cauhé MD, Dermatologist,

Dermatology Department, Hospital Universitario Ramón y Cajal, Madrid, Spain

Daniel Ortega-Quijano MD, Dermatologist, Dermatology Department, Hospital Universitario Ramón y Cajal, Madrid, Spain

Pedro Jaén-Olasolo MD, PhD, Chief, Dermatology Department, Hospital Universitario Ramón y Cajal, Madrid, Spain

Competing interests: None.

Funding: None.

Provenance and peer review: Not commissioned, externally peer reviewed.

\section{References}

1. Fisher BK, Dvoretzky I. Idiopathic calcinosis of the scrotum. Arch Dermatol 1978;114:957. doi: 10.1001/ archderm.1978.01640180089027.

2. Akinboro AO, Onilede DA, Babatunde TO, Oiwoh SO, Suleiman OA, Olabode OP. Idiopathic scrotal calcinosis: Report of 2 cases, and review of pathogenesis and factors that determine patients acceptance of surgical treatment. Clin Cosmet Investig Dermatol 2018;11:333-37. doi: 10.2147/ CCID.S142101.
3. Shapiro L, Platt N, Torres-Rodriguez VM. Idiopathic calcinosis of the scrotum. Arch Dermatol 1970;102(2):199-204.

4. Solanki A, Narang S, Kathpalia R, Goel A. Scrotal calcinosis: Pathogenetic link with epidermal cyst. BMJ Case Rep 2015. doi: 10.1136/bcr-2015211163.

5. Dubey S, Sharma R, Maheshwari V. Scrotal calcinosis: Idiopathic or dystrophic? Dermatol Online J 2010;16(2). doi: 10.1136/bcr-2015-211163.

6. Shah $\mathrm{V}$, Shet $T$. Scrotal calcinosis results from calcification of cysts derived from hair follicles: a series of 20 cases evaluating the spectrum of changes resulting in scrotal calcinosis. Am J Dermatopathol 2007;29(2):172-75. doi: 10.1097/01. dad.0000246465.25986.68

7. Meissner M, Kleemann J, Kaufmann R, Ochsendorf F. The erbium:YAG laser: A new technique for the treatment of scrotal calcinosis. J Eur Acad Dermatol Venereol 2017;31(4):207-09. doi: 10.1111/jdv.13963.

correspondence ajgp@racgp.org.au 